\title{
Aleitamento materno e desenvolvimento cognitivo
}

\section{Prezado Editor,}

Em dois artigos, publicados recentemente nesse prestigioso jornal ( $O$ aleitamento materno na prática clínica Giugliani ERJ; 2000:76:S238-S252, e Rede nacional de bancos de leite humano do Brasil: tecnologia para exportarGiugliani ERJ; 2000:78:183-4), a autora comemora a correlação positiva entre o tempo ou a presença de aleitamento materno com o desenvolvimento cognitivo. Nesses artigos, sublinha-se que "crianças amamentadas tinham escores de desenvolvimento cognitivo significativamente maiores do que crianças alimentadas com fórmula". Também ressalta-se que essa vantagem persiste até a adolescência. Em ambos os artigos, essa afirmação baseia-se unicamente no estudo de Anderson et al. ${ }^{1}$, reportado como uma meta-análise criteriosamente elaborada.

Alguns pontos, no entanto, devem ser ressaltados. Primeiro, as meta-análises, apesar de contribuírem efetivamente para o esclarecimento de pontos obscuros da literatura médica, principalmente referente a ensaios clínicos randomizados, apresentam limitações que se ampliam quando estudos observacionais são cotejados, particularmente no que se refere à heterogeneidade entre os mesmos ${ }^{2,3}$. Essa meta-análise de estudos observacionais, utilizada como referência única so- 
bre o tema, apresenta essas limitações e ainda falhas metodológicas explicitadas por Gordon ${ }^{4}$. Portanto, não seria tão criteriosamente desenvolvida como se poderia imaginar.

Segundo, a presença de uma associação ou correlação, nesse caso entre tempo de aleitamento e desenvolvimento cognitivo, não sustenta isoladamente pontes de causalidade; essas necessitam obrigatoriamente de uma base explicativa que as valide. $\mathrm{O}$ artigo de Anderson et al. ${ }^{1}$ cita presença de altas concentrações de ácidos graxos poliinsaturados de cadeia longa no leite materno, os quais seriam fundamentais para o bom desenvolvimento neurológico do lactente. Contudo, essa base explicativa é no mínimo frágil, tendo sido refutada em outros estudos ${ }^{5-7}$.

Terceiro, a não citação de estudos que apresentam resultados discordantes ao artigo de Anderson et al. ${ }^{1}$ introduziu um importante viés na seleção das evidências sobre esse tema. Além disso, a diferença geral de 3,2 pontos no escore cognitivo favorecendo as crianças alimentadas com leite materno, indicada por Anderson et al. ${ }^{1}$, carece de discussão sobre sua representação clínica. Portanto, considerações sobre o impacto clínico dessa diferença e a citação de artigos apontando resultados discordantes aos de Anderson et al. ${ }^{1}$ enriqueceriam o debate sobre esse ponto tão polêmico ${ }^{8-10}$.

Quarto, em nossa sociedade altamente competitiva, mesmo sem uma definição adequada, o termo "inteligência" tornou-se o jargão de referência para denominar os mais qualificados. Nesse sentido, questões envolvendo inteligência relacionam-se com sucesso, fracasso, obstáculos e discriminação. Considerando esses pontos, pergunta-se: qual seria o impacto dessa informação sobre a vida de um recém-nascido que não pode ser amamentado? Qual seria a consequiência de carregar a mácula de estar condenado a ser menos inteligente do que poderia ter sido? Todas essas dúvidas têm sido sustentadas por um grupo de evidências contraditórias.

Finalmente, as intervenções e estratégias objetivando o aumento das taxas de aleitamento materno vêm apresentando resultados significativos no Brasil nos últimos 20 anos. Fortes evidências apontam que o tempo de aleitamento materno correlaciona-se positivamente com a probabilidade de sobrevida de recém-nascidos, além de qualificá-la substancialmente. Portanto, a prática do aleitamento justifica-se plenamente, não necessitando de uma lógica circular construída para defender seu incentivo à margem da complexidade desse tema, o que poderia causar mais danos do que benefícios.

\section{Referências bibliográficas}

1. Anderson JW, Jonhstone BM, Remley DT. Breast-feeding and cognitive development: a meta-analysis. Am J Clin Nutr 1999;70:525-35.

2. Blettner M, Saurbrei W, Schlehofer B, Scheuchenpflug T, Friedenrich C. Traditional reviews, meta-analyses and pooled analyses in epidemiology. Int J Epidemiol 1999;28:1-9.

3. Egger M, Schneider M, Davey Smith G. Spurious precision? Meta-analysis of observational studies. BMJ 1998a; 316:61-6.

4. Gordon A. Breast-feeding, breast-milk feeding and intelligence quotient. Am J Clin Nutr 2000;72:1063-4.
5. Neuringer M, Reisbick S, Janowsky J. The role of n-3 fatty acids in visual and cognitive development: current evidence and methods of assessment. J Pediatr 1994;125:S44.

6. Scott DT, Janowsky JS, Carroll RE, Taylor JA, Auestad N, Montalto MB. Formula supplementation with long-chain polyunsaturated fatty acids: are there developmental benefits? Pediatrics 1998;102:E59.

7. Heird WC. The role of polyunsaturated fatty acids in term and preterm infants and breastfeeding mothers. Pediatr Clin North Am 2001;48:173-88.

8. Krugman S, Law P. Breastfeeding and IQ. Pediatrics 1999; 103:193.

9. Jain A, Concat J, Leventhal JM. How good is the evidence linking breastfeeding and intelligence? Pediatrics 2002; 109:1044-53.

10. Drane DL, Logemann JA. A critical evaluation of the evidence on the association between type of infant feeding and cognitive development. Paediatr Perinat Epidemiol 2000; 14:349-56.

\section{Prof. Dr. Marcelo Zubaran Goldani}

Mestre e Doutor em Pediatria pela Universidade de São Paulo. Professor do Dep. de Pediatria e Puericultura da Faculdade de Medicina da UFRGS

E-mail: mgoldani@hcpa.ufrgs.br

\section{Resposta da autora}

\section{Prezado Editor,}

Em resposta à carta do Prof. Dr. Marcelo Goldani, gostaria de fazer as seguintes considerações:

1. não existe consenso quanto à associação entre aleitamento materno e desenvolvimento cognitivo. Portanto, as opiniões divergem e a discussão em torno do tema é desejada e salutar;

2. o desenvolvimento cognitivo de um indivíduo é um processo complexo e influenciado por diversos fatores de ordem genética e ambiental, que interagem entre si. $\mathrm{O}$ aleitamento materno deve ser considerado como apenas um (provável) destes fatores;

3. a maioria dos estudos publicados sobre o tema (quase $70 \%$ ), independentemente da qualidade metodológica, conclui que o aleitamento materno promove o desenvolvimento cognitivo ${ }^{1}$;

4. a afirmação, em artigos citados pelo Dr.Goldani, de que "crianças amamentadas tinham escores de desenvolvimento cognitivo significativamente maiores do que crianças alimentadas com fórmula" foi baseada numa metaanálise que resume os resultados de estudos publicados até $1996^{2}$. Apesar de ser apenas uma referência bibliográfica, ela é baseada no resultado de 20 estudos (número de estudos selecionados para a meta-análise), portanto, com um peso bem maior do que uma simples referência. A referência, na realidade, era uma síntese do que tinha sido publicado até então. Outros estudos foram publicados após a data da publicação da referida meta-análise, refor- 
çando a existência da associação em questão. Apenas como exemplo, podemos citar um estudo de uma coorte que incluiu todos os nascimentos de um ano inteiro na cidade de Pelotas, $\mathrm{RS}^{3}$, e um estudo dinamarquês que encontrou uma associação entre inteligência e duração do aleitamento materno em adultos jovens, publicado no JAMA, em 2002 ${ }^{4}$;

5. a referida meta-análise foi publicada em uma revista médica de prestígio internacional (American Journal of Clinical Nutrition). A meta-análise se baseou em estudos observacionais, uma vez que ensaios clínicos randomizados inexistem por questões éticas. Para compensar essa limitação, modelos estatísticos foram cuidadosamente utilizados no sentido de minimizar a heterogeneidade dos resultados dos diferentes estudos;

6. o fato de não haver uma base explicativa que valide uma associação não quer dizer que tal associação não exista. Um exemplo atual é a associação entre uso de chupeta e menor duração do aleitamento materno. Tal associação foi confirmada em praticamente todos os estudos que a investigaram; no entanto, ainda não existe uma explicação plausível para a mesma. Outro exemplo é a associação entre anemia por deficiência de ferro e desenvolvimento mental ${ }^{5}$. Há especulações sobre os mecanismos envolvidos na possível associação entre aleitamento materno e melhor desenvolvimento cognitivo. Alguns defendem a presença de substâncias no leite materno que otimizariam o desenvolvimento cerebral ${ }^{5,6}$; outros acreditam que, além dos fatores nutricionais do leite materno, fatores comportamentais ligados ao ato de amamentar e à escolha do modo de como alimentar a criança estão envolvidos na associação em questão ${ }^{4}$;

7. o efeito do aleitamento materno no desenvolvimento cognitivo relatado na literatura pode não ser clinicamente relevante no nível individual; no entanto, no nível populacional esse efeito pode ser significativo para a socieda$\mathrm{de}^{7}$. Considerando não apenas o valor absoluto do QI, mas a prevalência de QI abaixo de 90 (limite inferior usualmente utilizado), Mortensen et al. encontraram $28 \%$ de adultos jovens amamentados por um mês, ou menos, com QI abaixo de 90, e 4\% entre os amamentados por mais de 9 meses $^{8}$. Existe a possibilidade, portanto, de que as crianças amamentadas tenham uma prevalência menor de QI < 90, e esse fato é relevante;

8. os resultados dos estudos científicos devem ser divulgados para que os indivíduos façam as suas opções após receberem todas as informações disponíveis. Não seria justo, nem ético, omitir informações por que elas podem gerar culpa ou desconforto em algumas pessoas. Não podemos, por exemplo, deixar de informar a uma gestante fumante que o cigarro pode trazer danos ao feto, por mais culpa que essa informação possa causar na fumante, que não consegue abandonar o hábito de fumar;

9. as informações são importantes não apenas para as mães e as famílias, mas também para os profissionais de saúde e os administradores. Por exemplo, alguns estudos têm mostrado que os prematuros são os que mais se beneficiam com o leite humano sob o ponto de vista cognitivo ${ }^{2,9}$.
Sabemos também das dificuldades encontradas pelas mães na amamentação desses bebês. Se tal vantagem for confirmada, cabe à sociedade em geral, e aos profissionais de saúde em especial, garantir leite humano para esta população, ou através de suas mães, quando possível e desejado, ou através de bancos de leite;

10. discordo da supervalorização de uma possível relação entre aleitamento materno e inteligência com o intuito de promover o aleitamento materno. A mídia, em algumas ocasiões, tem feito sensacionalismo sobre o fato. Até o momento, o que podemos afirmar (e transmitir para os profissionais de saúde e leigos) é que a maioria dos estudos mostra uma vantagem das crianças amamentadas sob o ponto de vista cognitivo; mas que tal fato ainda não está comprovado. Estudos mais criteriosos são necessários. Temos também que informar que o aleitamento materno é apenas um (provável) dos fatores envolvidos no desenvolvimento cognitivo, e que não se sabe a sua magnitude e importância;

11. é possível que novos estudos não confirmem o que a maioria dos estudos feitos até o momento têm mostrado. É possível também que a magnitude da diferença cognitiva entre as crianças amamentadas e não amamentadas seja bem maior do que a que tem sido relatada. Não há estudos que tenham comparado, por exemplo, crianças amamentadas segundo as recomendações atuais (dois ou mais anos, sendo os primeiros 6 meses de amamentação exclusiva) e as não amamentadas;

12. concluindo, há fortes indícios na literatura, porém não conclusivos, de que o aleitamento materno promove o desenvolvimento cognitivo. Os mecanismos envolvidos nessa possível associação ainda não estão esclarecidos (muito embora se saiba que a espécie humana durante praticamente toda a sua evolução contou com o leite materno e o ato de amamentar para chegar ao estágio em que se encontra). Estudos randomizados não são viáveis sob o ponto de vista ético, o que dificulta a comprovação (ou não) dessa associação. A população tem o direito de receber informações precisas, sem distorções ou exageros, de pesquisas cujos resultados possam influenciar nas suas decisões.

\section{Referências bibliográficas}

1. Jain A, Concat J, Leventhal JM. How good is the evidence linking breastfeeding and intelligence? Pediatrics 2002; 109:1044-53.

2. Anderson JW, Jooonstone BM, Remley DT. Breast-feeding and cognitive development: a meta-analysis. Am J Clin Nutr 1999; 70:525-35.

3. Halpern R, Giugliani ERJ, Victora CG, Barros FC, Horta BL. Fatores de risco para suspeita de atraso no desenvolvimento neuropsicomotor aos 12 meses de vida. J Pediatr (Rio J) 2000; 76(6):421-8.

4. Mortensen EL, Michaelsen KF, Sanders SA, Reinisch. The association between duration of breastfeeding and adult intelligence. JAMA 2002; 287:2365-71. 
5. Uauy R, Peirano P. Breast is best: human milk is the optimal food for brain development. Am J Clin Nutr 1999;70:433-4.

6. Lucas A, Morley R, Lister G, Lesson-Payne C. Breast milk and subsequent intelligence quotient in children born preterm. Lancet 1992;339:261-4.

7. Reynolds A. Breastfeeding and brain development. Pediatr Clin North Am 2001;48:159-71.

8. Mortensen EL. Breastfeeding ad later intelligence (Reply). JAMA 2002; 288:829.
9. Drane DL, Logemann JA. A critical evaluation of the evidence on the association between type of infant feeding and cognitive development. Paediatr Perinat Epiodemiol 2000;14:349-56.

\section{Dra. Elsa Giugliani}

Professora do Dep. de Pediatria e Puericultura da Universidade Federal do Rio Grande do Sul

E-mail: elsag@vortex.ufrgs.br 